\title{
The Design of Wireless Charging Device
}

\author{
Jian Chu ${ }^{1, a^{*}}$, Liqing Geng ${ }^{2, b^{*}}$, Huaijie Huang ${ }^{3, c}$ \\ ${ }^{1}$ Tianjin Key Laboratory of Information Sensing and Intelligent Control, Tianjin University of \\ Technology and Education, Tianjin, 300222, China \\ 2 Tianjin University of Technology and Education, Tianjin, 300222, China \\ ${ }^{3}$ Tianjin University of Technology and Education, Tianjin, 300222, China \\ achujian6@126.com , bgenglq2004@126.com, ${ }^{\mathrm{c}} 793887543 @ q q . c o m$
}

Keywords: Magnetic coupling resonance; Wireless charging; Transmission efficiency

Abstract. In recent years, the wireless power transmission is a new transmission mode. The wireless charging theory of magnetic coupling resonant mode is researched. The wireless charging device based on MCU is designed which includes sending unit and receiving unit. The sending unit is consists of resonant circuit, power supply, MCU control circuit, compensation capacitance, coupling coil, keyboard circuit and display circuit. The receiving unit is consists of coupling receiver coil, filter rectifier circuit, MCU control circuit, liquid crystal display circuit, voltage detection circuit, compensation capacitor and load. Using this system, mobile phone charging experiment was carried out. The experimental results show that the device realized the function of wireless charging.

\section{Introduction}

Wireless power transmission is transmitted by the electromagnetic field or electromagnetic waves[1-2]. Wireless charging supplies power for supply equipment by non-contact power. Comparing with the traditional way of cable power supply, electric spark produced between charging equipment and other unsafe factors such as the conductor exposed outside, wear and tear. Wireless charging technology has also changed "the disadvantages of a line charge", greatly improving the mobility of the electrical equipment. The wireless charging theory of magnetic coupling resonant mode is researched. The wireless charging device based on MCU is designed which includes two parts of sending and receiving. The primary coil produces alternating magnetic field in the sending part. Secondary coil produces alternating current in the receiving part and charging the battery for external equipment. The wireless charging function is realized. The voltage and current value of system can be detected in time and displayed on the LCD screen.

\section{The Basic Principle of Magnetic Coupling Resonance}

When ac current switch on a coil, alternating magnetic field is produced. The another coil near will generate induced electromotive force, so as to produce induced current. The above phenomenon is called the magnetic coupling. Magnetic coupling resonance principle diagram is shown in Fig.1. The sending terminal is comprised of the ac power Vs, sending coil L1 and harmonic compensation capacitor $\mathrm{C} 1$. The receive terminal is comprised of resonant coil L2, compensation capacitor $\mathrm{C} 2$ and load. Generally coil L1 and L2, harmonic compensation capacitor C1 and C2 parameters are the same. The electricity transmission distance between the sending coil and receiving coil is be defined as $\mathrm{d}$.

When the system works, ac current switch on sending coil, alternating magnetic field is produced. The induced current will be generated when the receiving coil is closed to sending coil which power over the load RL. The non-contact power supply is realized. The magnetic coupling equivalent circuit is shown in Fig.2[3-4]. 


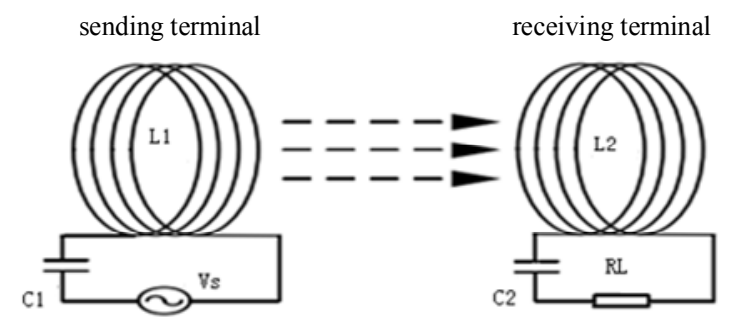

Fig. 1 The magnetic coupling. magnetic coupling resonance principle diagram

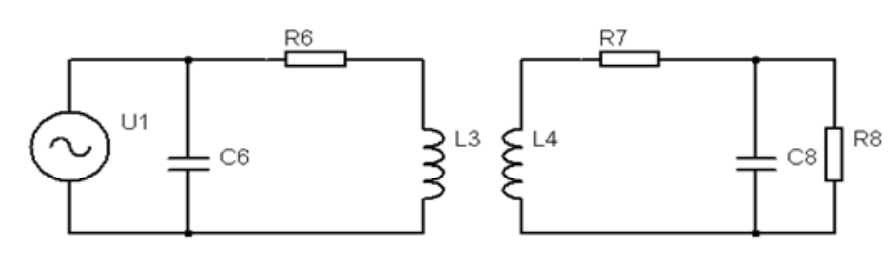

Fig. 2 The magnetic coupling equivalent circuit

In the diagram $R_{6}$ and $R_{7}$ are parasitic resistance, $C_{6}$ and $C_{8}$ are resonant capacitance , $R_{8}$ is load, $M$ is supposed to the mutual inductance of two coil, D is supposed to the distance of two coil. Assuming that the angular frequency of sending coin current is $\omega_{\text {and }}$ the Valid values is $I_{3}$ .When two coils are in a state of resonance, then

$$
C_{6}=\frac{L_{3}}{\omega^{2} L_{3}^{2}+R_{6}^{2}}
$$

)

$$
C_{8}=\frac{L_{4}}{\omega^{2} L_{4}^{2}+R_{4}^{2}}
$$

)

By KCL and KVL theorem, the following formula are deduced .

$$
I_{8}+\frac{R_{8} I_{8}}{1 / j \omega C_{8}}=I_{4}
$$

)

$$
I_{4}\left(j \omega L_{4}+R_{7}+\frac{1}{j \omega C_{8}+1 / R_{8}}\right)=j \omega M I_{3}
$$

)

From the above formula, effective current of load and output power can be formulated as:

$$
I_{8}=\frac{\omega M I_{3} \sqrt{\omega^{2} L_{4}{ }^{2}+R_{7}}}{\omega^{2} L_{4}{ }^{2}+R_{7}^{2}+R_{8} R_{7}}
$$

$$
P_{0}=I_{8}^{2} R_{8}
$$

)

Then, the efficiency of the system $\eta$ can be formulated as:

$\eta=\frac{P_{0}}{P_{0}+P_{1}+P_{2}}$

)

where, $P_{1}$ and $P_{2}$ are the loss power of sending coin and receiving coin. 


$$
\begin{aligned}
& P_{1}=I_{3}^{2} R_{6} \\
& P_{2}=I_{4}^{2} R_{7} \\
&
\end{aligned}
$$

The efficiency $\eta$ is higher, the power transmission is the greater. When the driving frequency is in accordance with the resonance frequency of the coil, the transmission efficiency $\eta_{\text {is }}$ the largest. When the driving frequency deviate from the resonance frequency of the coil, the transmission efficiency will decrease, thus the power transmission of electric is affected.

\section{The Design of Wireless Charging Device System}

The system of wireless electric energy transmission is composed of sending unit and receiving unit(Fig.3). The sending unit is consists of power converting circuit, syntonic circuit, AT89C52 MCU control circuit, keyboard circuit and LED display circuit etc. The AT89c52 MCU can output adjustable PWM signal to drive MOS tube which makes the LC resonance circuit to produce sine wave. Consequently, the sending coil produces an alternating current. Duty ratio can be adjusted though keyboard. The receiving unit is consists of rectifier bridge, STC12C5A60S2 MCU control circuit, voltage regulator circuit, voltage and current sampling circuit, LCD circuit and charging equipment etc. The receiving coil induces magnetic field to produce induced current which is stabilized though rectifier filter. After stable voltage charges their phone. At the same time, the voltage and current value are displayed on the LCD screen.
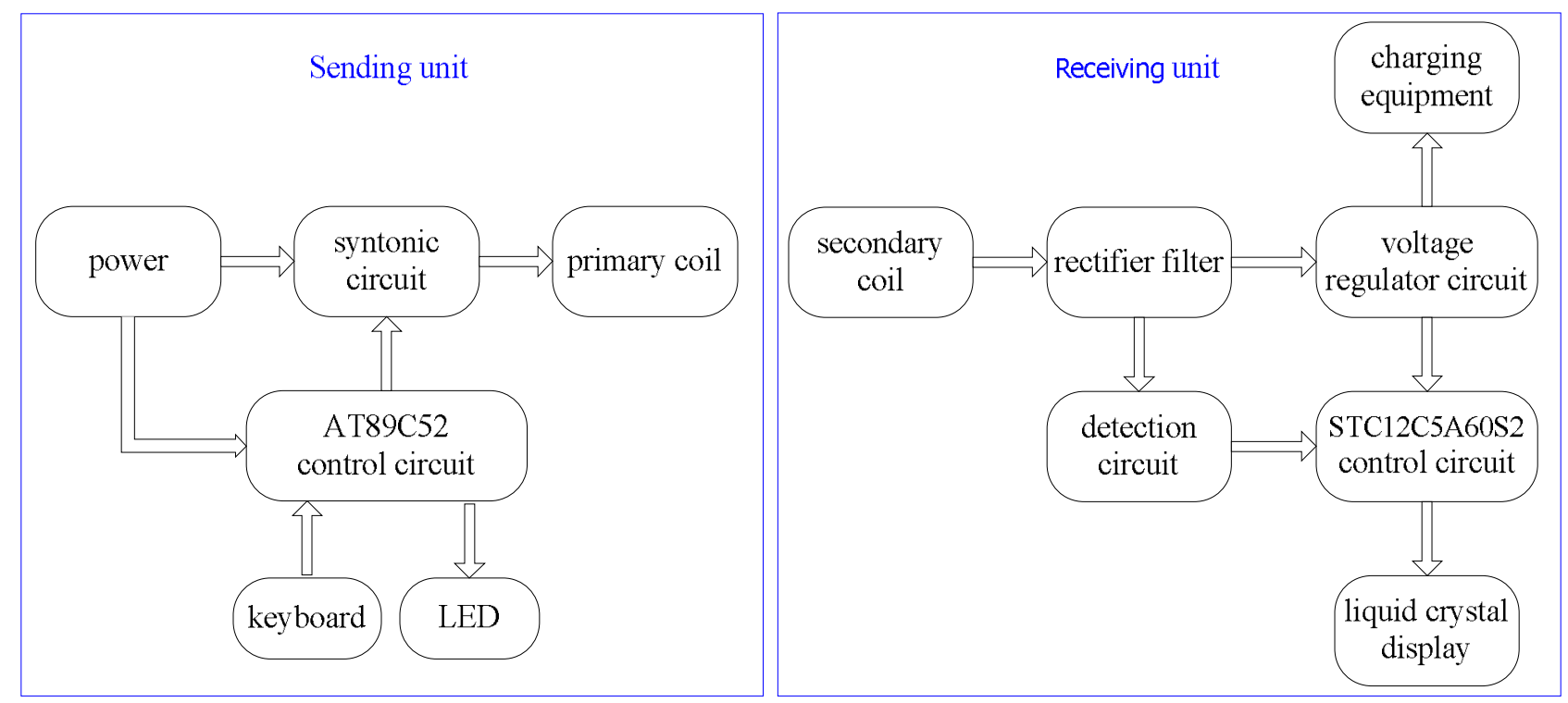

Fig.3 The system composition block diagram

\section{The Coil Resonant Inductance}

The inductance of coil mainly depends on the number of turns, winding method and magnetic core material. The number of coil turns is the more, the inductance of coil winding is greater. The quality factor Q of the coil are main parameters used to measure quality of coil. The size of the Q value shows the size of the coil loss. The $\mathrm{Q}$ value can be formulated as:

$$
Q=\frac{2 \pi f L}{R}
$$


When $\mathrm{L}$ value is unchanged, Q value is proportional to the coil itself resistance. The $\mathrm{Q}$ value of the coil is the higher, the loss of the circuit is the smaller. So the efficiency of the circuit is the higher。

The inductance of close winding toroidal inductor is formulated as[5-6]:

$$
L=N^{2} r u_{0}\left[\ln \left(\frac{8 r}{a}\right)-1.75\right]
$$

where, $\mathrm{N}$ is the coil number of turns, $\mathrm{r}$ is the coil radius, $\mathrm{a}$ is conductor section radius, $\mu 0$ is vacuum magnetic derivative.

\section{The Coupling Coefficient}

The hollow coil of coaxial parallel is used in the system. The coefficient of mutual induction between two resonance coil is formulated as:

$$
M=\frac{\pi}{4} \frac{u_{0} N_{1} N_{2}\left(r_{1} r_{2}\right)^{2}}{D^{3}}
$$

where, D is the distance of solenoid, $N_{1}$ is the sending coil number of windings, $N_{2}$ is the receiving coil number of windings, $r_{1}$ is the radius of sending coil twining, $r_{2}$ is the radius of receiving coil twining.

The coupling coefficient of two resonance coil is formulated as:

$$
k=k_{12}=k_{21}=\frac{\omega M}{2 \sqrt{L_{1} L_{2}}}
$$

where, $L_{1}$ is the inductance of sending coil, $L_{2}$ is the inductance of receiving coil.

\section{The Calculation of Resonance Capacitive}

The capacitive value is formulated as:

$$
C=\frac{1}{4 \pi^{2} f^{2} L}
$$

According to the above formula, the system adopts $0.2 \mathrm{~mm}$ concentrically wound coil to twine 34 circles whose diameter is $15 \mathrm{~cm}$. The inductance value calculated is $34 \mathrm{uH}$. When $6 \mathrm{KHZ}$ frequency signal is outputted by MCU, the capacitance value calculated is $104 \mathrm{PF}$.

\section{The Design of System Software}

\section{The Design of Sending Unit Software}

The main program chart of sending unit is shown in Fig.4. The main function is to output PWM signal and adjust key to suitable gears which is displayed on LED.

\section{The Design of Receiving Unit Software}

The main program chart of receiving unit is shown in Fig.5. The main function is to acquire the value of electric current and voltage which are displayed on LCD. 


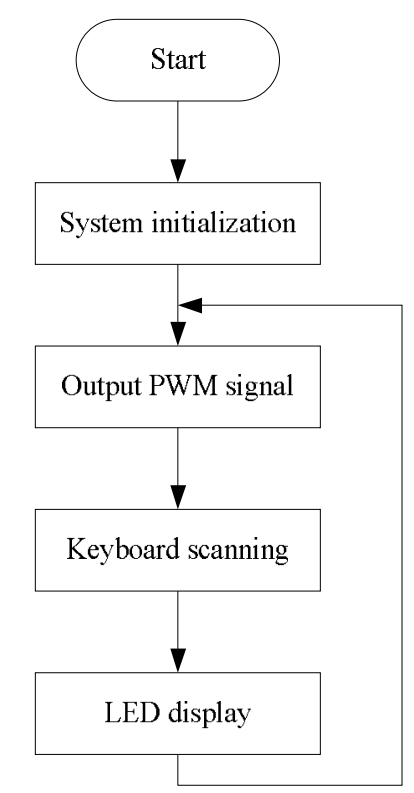

Fig.4 The main program of sending uni $t$

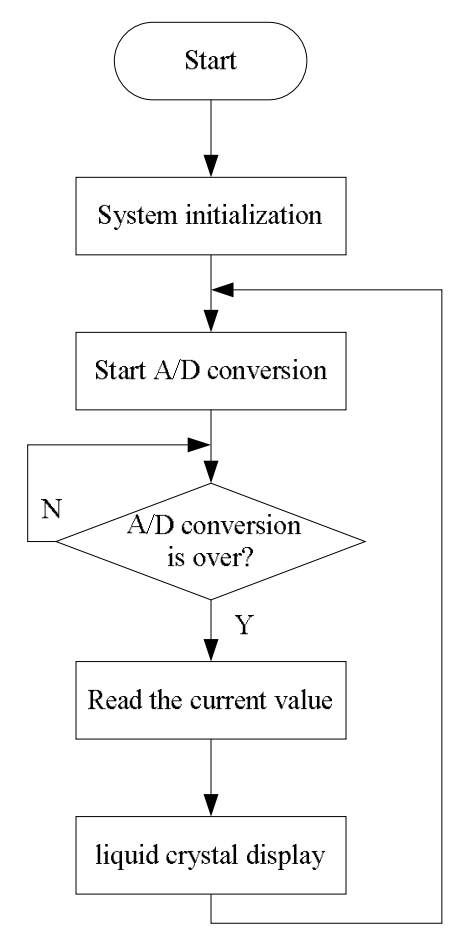

Fig.5 The main program of receiving unit

\section{Conclusions}

This paper studied on wireless charging principle. The wireless charging device of magnetic coupling resonant mode is designed. The device is supplied by $24 \mathrm{~V}$ DC power supply. The AT89c52 MCU can output adjustable PWM signal to drive MOS tube which makes the alternating current which is rectified and filtered. The mobile phone is charged though USB after voltage stabilization. The electric current and voltage value are acquired which are displayed on LCD. The result of the system test shows that the device realizes the function of mobile phone charging. The transmission efficiency of the system is about $40 \%$. The feasibility and effectiveness of the system and verified.

\section{References}

[1] Zhixiong Wang, Jinglei Hu, Jun Liang, Changhua Wang, " Application Prospect of Wireless Electric Power Transmisson Technology," Journal of Air Force Engineering University(Natural Science Edition,2003,4(1).83-85.(in Chinese)

[2] Guankai Li, Haifeng Liang, Chengyong Zhao, Ming Zhou, Gengyin Li, "Comparison and prospect of several special technologies of power transmission," Electric Power, 2004,37(4).43-48. (in Chinese)

[3]Tan L L, Huang X L, Huang H, et al. Transfer efficiency optimal control of magnetic resonance coupled system of wireless power transfer based on frequency control. Sci China Tech Sci, 2011, 54: 1428-1434. (in Chinese)

[4] Wen-zhen Fu, Bo Zhang, Dong-yuan Qiu, Wei Wang," Maximum Efficiency Analysis and Design of Self-resonance Coupling Coils for Wireless Power Transmission System", Proceedings of the CSEE,2009,28(18):21-26. (in Chinese)

[5] Zhihao Qin, Shiqin Jiang," The Mutual Inductance Between Circle and Disk and The Analysis of Magnetic Flux Density," COMPUTER KNOWLEDGE AND TECHNOLOGY, 2008 4(28):15-17. (in Chinese)

[6] Linan Qu,"Study on the Mechanism of Wireless Power Transfer Based on Magnetic Resonances," HARBIN INSTITUTE OF TECHNOLOGY,2010:20-23. (in Chinese) 


\section{Acknowledgments}

Fund program: Natural Science Foundation of China (61178081). Natural Science Foundation of Tianjin (14jczdjc3630). Beforehand research project for schools (jk14-13). 